\title{
Effects of the Radial Extracorporeal Shock Wave Therapy (rESWT) in Patients With Calcific Tendinopathy of the Shoulder
}

\author{
Dragana Dragičević-Cvjetković1,2, Tatjana Erceg-Rukavina', Slavko Manojlović²
}

\section{ABSTRACT}

Introduction: Calcific tendinopathy (CT) of the shoulder is frequently a painful condition that is occurring when there are calcium deposits in the tendons of the rotator cuff. There are many options for treatment starting from therapeutic options like medicaments, physical therapy and radial extracorporeal shock wave therapy (rESWT). If conservative treatment fails, surgical treatment is the next option. The purpose of this study was to investigate the outcome effect of rESWT in patients with calcific tendinopathy.

Methods: A prospective study from February 2010 to March 2019 monitored 67 patients with CT of the shoulders of the average age of $47.06 \pm 15.2$ (mean \pm standard deviation) who were treated with a rESWT. All patients were evaluated clinically with selected radiographic evaluation of the shoulder before therapeutic intervention. The treatment protocol consisted of a sound pressure intensity of 3 bar, a wave frequency of $14 / \mathrm{sec}$, a total of 2,000 waves per session. All patients performed a treatment consisting of three sessions described every seven days. The outcome parameters were VAS scale of pain and shoulder radiography before and after therapy. The Student's t-test was used in the statistical analysis.

Results: Clinical and radiographic improvement was recorded in $70 \%$ of patients in the treatment of CT of the shoulder 6 weeks after the therapy was performed with a rESWT $(\mathrm{p}<0.05)$ in terms of reduced pain and disintegration of calcification.

Conclusion: rESWT has showed positive effects in the treatment of patients that have calcific tendinopathy of the shoulder.

Key words: rESWT, calcific tendinopathy, shoulder.

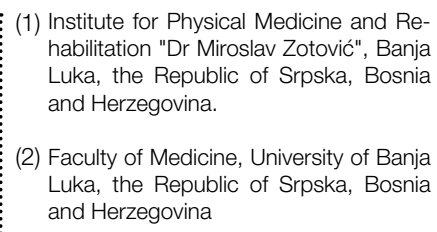

1) Institute for Physical Medicine and Rehabilitation "Dr Miroslav Zotović", Banja Luka, the Republic of Srpska, Bosnia and Herzegovina.

(2) Faculty of Medicine, University of Banja Luka, the Republic of Srpska, Bosnia and Herzegovina

Correspondence:

DRAGANA DRAGIČEVIĆ

- CVJETKOVIĆ

E: dragicevicdr@gmail.com

M: +38765 711138

\section{ARTICLE INFO}

Received: 22 July 2019

Revision received: 2 September 2019 Accepted: 10 September 2019

\section{INTRODUCTION}

Calcific tendinopathy of the shoulder is often a painful condition that can be a major therapeutic challenge. This clinical entity represents one of the overuse syndrome, characterised by deposition of calcium deposits usually in the tendons of the rotator cuff (predominantly in supraspinatus muscle) with the possibility of propagation into a subchromial or subdeltoid spaces. ${ }^{1}$ Epidemiological studies suggest that this syndrome is most common in women aged 40-60. ${ }^{2}$ To confirm the diagnosis of calcific tendinopathy of the shoulders, most often, after a clinical ex- amination of the shoulder, radiographs, ultrasound and magnetic resonance are used. ${ }^{3}$ Radial extracorporeal shock wave therapy (rESWT) is one of the non-invasive treatment options for calcific tendinopathy of the shoulders with medication, physiotherapy and ultrasound-guided injection and needling. ${ }^{4}$ If conservative management fails, surgical intervention in the form of arthroscopy and debridement is considered. 5 Some of the authors estimate the effectiveness of rESWT in patients with CT at 78-91\%.6 The mechanism of action rESWT, which is still be- 
ing studied is triple: mechanical sound waves of different intensities lead to disintegration of calcite, the analgesic effect is achieved at the level of the 'gate control system' by the hyperstimulation analgesia and the direct effects of the sound flux in the treated region is caused by neovascularisation. ${ }^{7,8}$ Consensus on the treatment algorithm for this overload syndrome has not yet been achieved.

\section{AIM OF THE STUDY}

The purpose of this study was to investigate the outcome effect of rESWT in patients with CT in whom other conservative treatment methods did not give the expected results or improvement within 6 months of the onset of symptoms.

\section{METHODS}

In the period from February 2010 to March 2019, 67 patients with calcific tendinopathy of the shoulder were monitored by a prospective study. The research was carried out at the Institute of Physical Medicine and Rehabilitation 'Dr Miroslav Zotović' Banja Luka. A triage of patients for rESWT was performed by a physiatrist and orthopaedic surgeon during outpatient work at the Orthopaedic Department of the Institute, and after seeing the ineffectiveness of other methods of conservative treatment at last 6 months after the symptoms have been reported. After the clinical examination, all patients had radiographs of the shoulders in the AP position. The treatment consisted of three rESWT sessions that were conducted every 7 days with the following parameters: intensity of sound pressure 3 bar, frequency of waves 14 / sec, a total of 2000 waves per session. The treatment outcome parameters were VAS scaling pain and shoulder radiography before and after therapy. Patients determined the intensity of pain before and after rESWT using a visually analogue scale of pain (VAS). VAS scale of pain is a line of 10 units (from o to 10), where the mark is 0 - no pain and the 10 - worst possible pain. Other ratings on the scale are 2 and 3 - mild pain, 4 and 5 - moderate pain, 6 and 7 - severe pain, and 8 and 9 - very severe pain. The outcome of treatment of radiography was graded in three degrees: complete disintegration of calcification (complete healing), disintegration of calcifica- tion by more than 50\% (satisfactory result) and calcification without change (failed treatment). The follow-up period was 6 weeks after the last session of the rESWT when patients came for a control exam with control radiographs that were done on the same device as the initial device.

In the statistical analysis, the parameters of descriptive statistic (mean and standard deviation) and Student's t-test were used. As a level of statistical significance the value $\mathrm{p}<0.05$ was taken.

\section{RESULTS}

Regarding clinical data relevant to this study, it is important that all monitored patients with CT are included in rESWT after realising that other conservative treatment methods have not led to a subjective and clinical improvement. The investigated sample of patients consisted of 43 women and 24 men of the average age of 47.06 \pm 15.2 (Table 1 ).

Table 1: Distribution of patients with calcific tendinopathy of the shoulder treated with radial extracorporeal shock wave therapy (rESWT) by age and gender

\begin{tabular}{lll}
\hline Gender & Number & Age (mean \pm standard deviation) \\
\hline Male & 24 & $50.12 \pm 13.43$ \\
Female & 43 & $44.00 \pm 16.97$ \\
Total & 67 & $47.06 \pm 15.2$ \\
\hline
\end{tabular}

A statistically significant reduction in pain was recorded in all patients after 6 weeks of rESWT ( $\mathrm{p}<0.05$ ) (Figure 1).

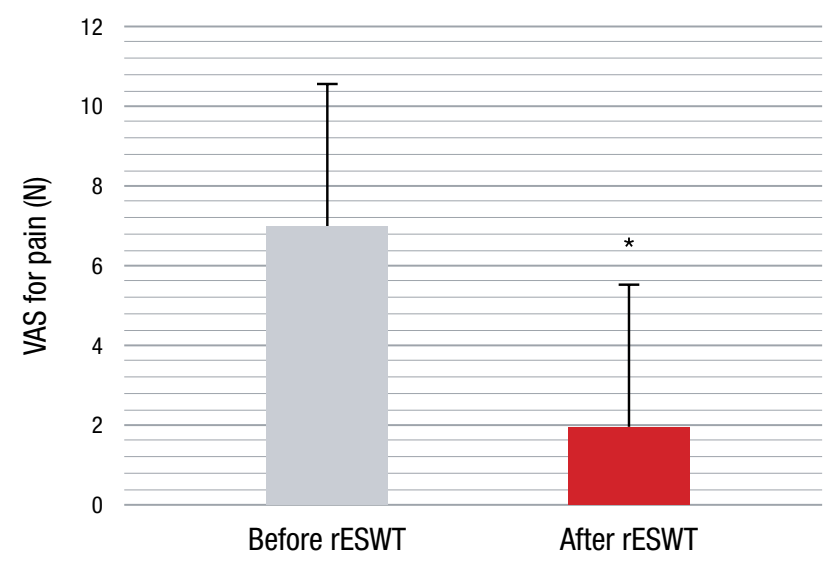

Figure 1: Outcome of treatment measured by mean values of visual analogue scale (VAS) for pain \pm SD before and after radial extracorporeal shock wave therapy (rESWT). * $p<0.05$ vs before rESWT. 


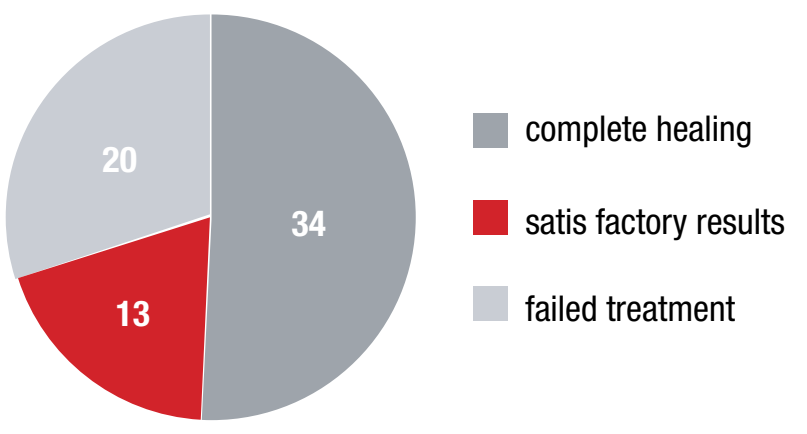

Figure 2: Radiographic outcomes of radial extracorporeal shock wave therapy (rESWT) - number of patients.

Figure 2 shows radiographic complete healing after using rESWT in a half of the patients, while the other $20 \%$ is at the level of a satisfactory result. In summary, in $70 \%$ of patients, a statistically significant positive effect of rESWT has been demonstrated $(\mathrm{p}<0.05)$.

\section{DISCUSSION}

This prospective study was followed by 67 patients aged 30 to 60 who were treated with rESWT due to calcific tendinopathy of the shoulder. Epidemiologically, in this case, it was confirmed that CT is most common at the middle-aged women. rESWT is effective shortterm treatment in patients with CT in clinically significant pain reduction and disintegration of calcification as the primary cause of damage to the shoulder function. Most of the public studies show positive outcomes for the treatment of CT by rESWT..$^{9-17}$ It is undeniable that there are also studies that have not proven that rESWT in CT treatment has a better effect than placebo. ${ }^{18-20}$

It is believed that shortcoming of this study is short period of follow-up, but knowing the pathophysiology of calcification in soft tissues, good results in a long-term can be expected. This is also testified by the research of other authors. ${ }^{21-24}$ A group of Italian authors has shown the effectiveness of shock wave therapy (SWT) as a decrease in pain and a significant rate of calcification disintegration in a six-month follow-up period in patients with CT ${ }^{25}$ Spindler et al estimated complete healing by rESWT in three patients after two years with complete disintegration of calcite immediately after treatment. 26 Long-term follow-up studies have shown positive effects of rESWT in almost 90\% patients with CT. These results are radiographically confirmed. No calcium deposits were recorded again in the rotator cuff tendons in the next two years after rESWT. It has been shown that the reduction of shoulder pain is directly related to the reduction/elimination of calcite. ${ }^{21}$ Rompe et al (1997) reported positive effects of rESWT in more than two-thirds of patients with shoulder $\mathrm{CT}$ and complete or partial disintegration of the calcite in slightly more than half of patients. ${ }^{27}$

In the second study, Rompe et al announced that shock waves therapy was no superior to surgery in patients with calcific tendinopathy of the shoulder. ${ }^{28}$

The effect of different doses of rESWT was not investigated or compared in relation to surgical treatment in this study. These could be the subjects of future research.

\section{CONCLUSION}

rESWT showed positive effects in the treatment of patients that have calcific tendinopathy of the shoulder. This option of treatment should be considered in cases where other conservative treatment methods do not show any signs of improvement.

\section{ACKNOWLEDGEMENTS}

None.

\section{CONFLICT OF INTEREST}

None.

\section{REFERENCES}

1. Sansone V, Maiorano E, Galluzzo A, Pascale V. Calcific tendionopathy of the shoulder: clinical perspectives into the mechanisms, pathogenesis and treatment. Orthop Res Rev 2018;10(1):63-72.

2. Tashjian RZ. Epidemiology, natural history, and indications for treatment of rotator cuff tears. Clin Sports Med 2012;31(4):589-604.

3. Greis AC, Derrington SM, McAuliffe M. Evaluation and nonsurgical management of rotator cuff calcific tendinopathy. Orthop Clin North Am 2015;46(2):293302.

4. Merolla G, Singh S, Paladini P, Porcellini G. Calcif- 
ic tendinitis of the rotator cuff: state of the art in diagnosis and treatment. J Orthopaed Traumatol 2016;17(1):7-14.

5. Hurt G, Baker CL. Calcific tendinitis of the shoulder. Orthop Clin North Am 2003;34(4):567-75.

6. Sems A, Dimeff R, Iannotti JP. Extracorporeal shock wave therapy in the treatment of chronic tendinopathies. J Am Acad Orthop Surg 2006;14(4):195-204.

7. Cacchio A, Paoloni M, Barile A, Don R, de Paulis F, Calvisi $\mathrm{V}$, et al. Effectiveness of radial shock-wave therapy for calcific tendinitis of the shoulder: single-blind, randomized clinical study. Phys Ther 2006;86(5):672-82.

8. Wang CJ., Wang FS., Yang KD. Shock wave therapy induces neovascularization at the tendon-bone junction. A study in rabbits. J Orthopaed Res 2003;21(6):984989.

9. Buch M, Knorr U, Fleming L, Theodore G, Amendola A, Bachmann C, et al. Extracorporeal shock wave therapy in symptomatic heel spurs. An overview. Orthopade 2002;31(7):637-44. German.

10. Perez M, Weiner R, Gilley JC. Extracorporeal shock wave therapy for plantar fasciitis. Clin Podi Med Surg 2003;20(2):323-34.

11. Roehrig GJ, Baumhauer J, DiGiovanni BF, Flemister AS. The role of extracorporeal shock wave on plantar fasciitis. Foot Ankle Clin 2005;10(4):699-712.

12. Strash WW, Perez RR. Extracorporeal shock wave therapy for chronic proximal plantar fasciitis. Clin Podi Med Surg 2002;19(4):467-76.

13. Thomson CE, Crawford F, Murray GD. The effectiveness of extra corporeal shock wave therapy for plantar heel pain: a systematic review and meta-analysis. BMC Musculoskelet Disord 2005;6:19.

14. Wilner JM, Strash WW. Extracorporeal shock wave therapy for plantar fasciitis and other musculoskeletal conditions utilizing the update. Clin Podi Med Surg. 2004;21(3):441-7.

15. Daecke W, Kusnierczak D, Loew M. Long-term effects of extracorporeal shock wave therapy in chronic calcific tendinitis of the shoulder. J Shoulder Elbow Surg 2002;11(5):476-80.

16. Hsu CJ, Wang DY, Tseng KF, Fong YC, Hsu HC, Jim YF. Extracorporeal shock wave therapy for calcific tendinitis of the shoulder. J Shoulder Elbow Surg 2008;17(1):55-9.

17. Pleiner J, Crevenna R, Langenberger H, Keilani M, Nuhr M, Kainberger F, et al. Extracorporeal shock wave treatment is effective in calcific tendonitis of the shoulder. A randomized controlled trial. Wien Klin Wochenschr 2004;116(15-16):536-41.
18. Buchbinder R, Green SE, Youd JM, Assendelft WJ, Barnsley L, Smidt N. Systematic review of the efficacy and safety of shock wave therapy for lateral elbow pain. J Rheum 2006;33(7):1351-63.

19. Buchbinder R, Ptasznik R, Gordon J, Buchanan J, Prabaharan V, Forbes A. Ultrasound-guided extracorporeal shock wave therapy for plantar fasciitis: a randomized controlled trial. JAMA 2002;288(11):1364-72.

20. Speed CA, Nichols D, Wies J, Humphreys H, Richards C, Burnet S, et al. Extracorporeal shock wave therapy for plantar fasciitis. A double blind randomized controlled trial. J Orthop Res 2003;21(5):937-40.

21. Wang CJ, Yang KD, Wang FS, Chen HH, Wang JW. Shock wave therapy for calcific tendinitis of the shoulder: a prospective clinical study with two-year follow-up. Am J Sports Med 2003;31(3):425-30.

22. Chou W, Wang C, Wu K, Yang Y, Ko J, Siu K. Prognostic factors for the outcome of extracorporeal shock wave therapy for calcific tendinitis of the shoulder. Bone Joint J 2017;99-B(12):1643-50.

23. Mouzopoulos G, Stamatakos M, Mouzopoulos D, Tzurbakis M. Extracorporeal shock wave treatment for shoulder calcific tendonitis: a systematic review. Skeletal Radiol 2007;36:803-811.

24. Kolk A, Auw Yang KG, Tamminga R, Van Der Hoeven H. Radial extracorporeal shock wave therapy in patients with chronic rotator cuff tendinitis: a prospective randomised double-blind placebo controlled multicentre trial. Bone Joint J 2013;95-B(11):1521-6.

25. Ioppolo F, Tattoli M, Di Sante L, Venditto T, Tognolo L, Delicata M, et al. Clinical improvement and resorption of calcifications in calcific tendinitis of the shoulder after shock wave therapy at 6 months' follow-up: A systematic review and meta-analysis. Arch Phys Med Rehabil 2013;94(9):1699-706.

26. Spindler A, Berman A, Lucero E, Braier M. Extracorporeal shock wave treatment for chronic calcific tendinitis of the shoulder. J Rheumatol 1998;25(6):1161-3.

27. Rompe JD, Eysel P, Hopf C, Krischek O, Vogel J, Bürger R, et al. Extracorporeal shock wave therapy in orthopedics. Positive results in tennis elbow and tendinitis calcarea of the shoulder. Fortschr Med 1997;115(18): 26, 29-33. German.

28. Rompe JD, Zoellner J, Nafe B. Shock wave therapy versus conventional surgery in the treatment of calcific tendinitis of the shoulder. Clin Orthopaed Rel Res 2001;387:72-82. 